\title{
A SURVEY ON THE EXISTENCE, UNIQUENESS AND REGULARITY QUESTIONS TO FULLY NONLINEAR ELLIPTIC PARTIAL DIFFERENTIAL EQUATIONS
}

\section{J. TYAGI AND R.B. VERMA}

Abstract. The aim of this survey paper is to provide the recent developments on the existence, uniqueness and regularity results to fully nonlinear elliptic equations of the form

$$
\left\{\begin{aligned}
F\left(x, u, D u, D^{2} u\right) & =f(x) \text { in } \Omega, \\
u & =0 \text { on } \partial \Omega,
\end{aligned}\right.
$$

where $\Omega$ is a smooth bounded domain in $\mathbb{R}^{n}$.

Mathematics subject classification (2010): 35A08, 35J60, 35J25, 49L25, 35B53.

Keywords and phrases: fully nonlinear elliptic equation, Bellman-Isaacs equation, viscosity solutions, Perron's comparison principle, Pucci extremal operator, singular elliptic equations, Monge-Ampère equation, eigenvalue problems.

\section{REFERENCES}

[1] S. ALARCÓN, L. ITURRIAGA AND A. QUAAS, Existence and multiplicity results for Pucci's operators involving nonlinearities with zeros, Cal. Vari. Part. Diff. Eq., 45, 3-4 (2012), 443-454.

[2] A. D. ALEKKANDROV, The Dirichlet problem for the equation Det $\left\|z_{i, j}\right\|=\phi\left(z_{1}, \ldots, z_{n}, z, x_{1}, \ldots, x_{n}\right)$ , Vestnik Leningrad. Univ., 1, 1 (1958), 5-24.

[3] D. ARAÚJO, E. V. TEIXEIRA, Geometric approach to nonvariational singular elliptic equations, Arch. Rational Mech. Anal., 209, 3 (2013), 1019-1054.

[4] S. N. ARMSTRONG, The Dirichlet problem for the Bellman equation at resonance, J. Differential Equations, 247, (2009), 931-955.

[5] S. N. ARMSTRONG, Principal eigenvalues and an anti-maximum principle for homogeneous fully nonlinear elliptic equations, J. Differential Equations, 246, (2009), 2958-2987.

[6] S. N. ARMSTRONG, Principal half-eigenvalues of fully nonlinear homogeneous elliptic operators, $\mathrm{PhD}$ thesis, University of California, Berkeley (2009).

[7] S. N. ARMSTRONG, L. E. SILVESTRE AND C. K. SMART, Partial regularity of solutions of fully nonlinear, uniformly elliptic equations, Commun. Pure Appl. Math, 65, 8 (2012), 1169-1184.

[8] S. N. ARMSTRONG, B. SIRAKOV, Nonexistence of positive supersolutions of elliptic equations via the maximum principle, Comm. PDE, 36, 11 (2011), 2011-2047.

[9] S. N. ARMSTRONG, B. SIRAKOV, Sharp Liouville results for fully nonlinear equations with powergrowth nonlinearities, Ann. Sc. Norm. Super. Pisa Cl. Sci., 10, 3 (2011),711-728.

[10] S. N. ARMSTRONG, C. K. SMART AND B. SIRAKOV, Fundamental solutions of homogeneous fully nonlinear elliptic equations, Comm. PDE, 64, 6 (2011), 737-777.

[11] S. N. ARMSTRONG, C. K. SMART AND B. SIRAKOV, Singular solutions of fully nonlinear elliptic equations and applications, Arch. Rational Mech. Anal., 205, 2 (2012), 345-394.

[12] I. Y. BAKE L' MAN, Geometric methods for solving elliptic equations, "Nauka", Moscow, 1965.

[13] M. BARDI, I. CAPUZZO-DOLCETTA, Optimal control and viscosity solutions of Hamilton-JacobiBellman equations, Modern Birkhauser Classics, (25) 2008.

[14] G. BARLES, Existence results for first order Hamilton Jacobi equations, Annales de $1^{\prime}$ institut Henri Poincaré (C) Analyse non linéaire, 1, 5 (1984), 325-340. 
[15] G. BARLES, A short proof of the $C^{0, \alpha}$-regularity of viscosity subsolutions for superquadratic viscous Hamilton-Jacobi equations and applications, Nonlinear Anal., 73, 1 (2010), 31-47.

[16] G. BARLES, A. PORRETTA, Uniqueness for unbounded solutions to stationary viscous HamiltonJacobi equations, Ann. Sc. Norm. Super. Pisa, Cl. Sci. 5, 5 (2006), 107-136.

[17] G. BARLES, P. E. SOUGANIDIS, Convergence of approximation schemes for fully nonlinear second order equations, Asymptotic Anal., 4, 3 (1991), 271-283.

[18] S. A. BELBAS, Weak solutions of the Hamilton-Jacobi-Bellman equation, Systems and Control Letters, 1, 3 (1981), 168-172.

[19] H. BERESTYCKI, L. NIRENBERG, On the method of moving planes and the sliding method, Boll. Soc. Brasil Mat., 22, 1 (1991), 237-275.

[20] H. BERESTYCKI, L. NIRENBERG, S. R. S. VARADHAN, The principal eigenvalue and maximum principle for second-order elliptic operators in general domains, Comm. Pure Appl. Math., 47, 1 (1994), 47-92.

[21] I. BIRINDELLI, F. DEMENGEL, Comparison principle and Liouville type results for singular fully nonlinear operators, Ann. Fac. Sci Toulouse Math, 132 (2004), 261-287.

[22] I. BIRINDELLI, F. DEMENGEL, First eigenvalue and maximum principle for fully nonlinear singular operators, Advances in Partial Diff. Equations, 11, 1 (2006), 91-119.

[23] I. BIRINDELLI, F. DEMENGEL, Eigenvalue, maximun principle and regularity for fully non linear homogeneous operators, Commun. Pure Appl. Anal., 6, 2 (2007), 335-366.

[24] I. BIRINDELLI, F. DEMENGEL, The Dirichlet problem for singular fully nonlinear operators, Discrete Contin. Dynam. Systems, Supplement, (2007), 110-121.

[25] I. BIRINDELLI, F. DEMENGEL, Eigenvalue and Dirichlet problem for fully-nonlinear operators in non-smooth domains, J. Math. Anal. Appl. 352, 2 (2009), 822-835.

[26] I. BIRINDELLI, F. DEMENGEL, Eigenfunctions for singular fully non linear equations in unbounded domain, NoDEA Nonlinear Differential Equations Appl., 17, 6 (2010), 1-30.

[27] H. BREZIS, L. C. EVANS, A variational inequality approach to the Bellman-Dirichlet equation for two elliptic operators, Arch. Rational Mech. Anal., 71, 1 (1979), 1-13.

[28] J. BUSCA, M. J. ESTEBAN AND A. QUAAS, Nonlinear eigenvalues and bifurcation problems for Pucci's operator, Annales de l'Institut Henri Poincare (C) Non Linear Analysis, 22, 2 (2005), 187 206.

[29] J. BUSCA, B. SIRAKOV, Harnack type estimates for nonlinear elliptic systems and applications, Annales de 1' Institut Henri Poincaré (C) Non Linear Analysis, 21, 5 (2004), 543-590.

[30] X. CABRÉ, Elliptic PDEs in probability and geometry, symmetry and regularity of solutions, Discr. Cont. Dyn. Sys., 20, 3 (2008), 425-457.

[31] X. CABRÉ, On the Alexandroff-Bakelman-Pucci estimate and the reversed Hölder inequality for solutions of elliptic and parabolic equations, Comm. Pure Appl. Math., 48, 5 (1995), 539-570.

[32] X. CABRÉ, L. A CAFFARELLI, Interior $C^{2, \alpha}$ regularity theory for a class of nonconvex fully nonlinear elliptic equations, J. Math. Pures Appl., 82, (2003), 573-612.

[33] L. A. CAFFARELLI, Interior $W^{2, p}$-estimates for solutions of the Monge-Ampère equation, Annals of Math., 131, 1 (1990), 135-150.

[34] L. A. CAFFARELLI, Elliptic second order equations, Rend. Sem. Mat. Fis. Milano, 58, (1988), 253 284.

[35] L. A. CAFFARELLI, Interior a priori estimates for solutions of fully non-linear equations, Annals of mathematics second series 130, 1 (1989), 189-213.

[36] L. A. CAFFARELLI, X. CABRÉ, Fully nonlinear elliptic Equations, Amer. Math. Soc., 43 (1995).

[37] L. CAFFARELLI, L. NIRENBERG, AND J. SPRUCK, The dirichlet problem for nonlinear secondorder elliptic equations. Part II. Complex Monge-Ampère, and uniformaly elliptic equations, Comm. Pure Appl. Math., 38, 2 (1985), 209-252.

[38] L. CAFFARELLI, L. NIRENBERG, AND J. SPRUCK, The Dirichlet problem for nonlinear second order elliptic equations, Part III: Functions of the eigenvalues of the Hessian, Acta. Math, 155, 1 (1985), 261-301.

[39] I. CAPUZZO DOLCETTA, A. CUTRÌ, Hadamard and Liouville type results for fully nonlinear partial differential inequalities, Commun. Contemp. Math., 5, 3 (2003), 435-448

[40] I. CAPUZZO DOLCETTA, A. VITOLO, On the maximum principle for viscosity solutions of fully elliptic equations in general domains, Le Matematiche 62, 2 (2007), 69-91.

[41] K.C CHANG, Methods in nonlinear analysis, Springer Monographs in Mathematics, 2005. 
[42] S.Y.CHENG, S.T. YAU, On the regularity of the Monge-Ampère equation $\operatorname{det}\left(\partial^{2} u / \partial x_{i} \partial x_{j}\right)=F(x, u)$, Comm. Pure Appl. Math., 30, 1 (1977), 41-68.

[43] F.C. CIRSTEA, C. TROMBETTI, On the Monge-Ampère equation with boundary blow-up: existence, uniqueness and asymptotics, Calc. Var. Partial Differ. Equ., 31, 2 (2008), 167-186.

[44] H.O. CORDES, Über die erste Randwertaufgabe bei quasilinearen Differentialgleichungen zweiter Ordnung in mehr als zwei Variablen, Math Ann. 131, 3 (1956), 278-312.

[45] M. G. CRANDALL, L. CAFFARELLI AND M. KOCAN, A. ŚWIECH, On viscosity solutions of fully nonlinear equations with measurable ingredients, Comm. Pure Appl. Math., 49, 4 (1996), 365-398.

[46] M. G. CRANDALL, L. C. EVANS, AND P. L. LIONS, Some properties of viscosity solutions of Hamilton-Jacobi equations, Trans. Amer. Math. Soc., 282, 2 (1984), 487-502.

[47] M. G. CRANDALL, H. ISHII AND P. L. LIONS, User's guide to viscosity solutions of second order partial differential equations, Bull. Amer. Math. Soc., 27, 1 (1992), 1-67.

[48] M. G. CRANDALL, H. ISHII, AND P. L. LIONS, Uniqueness of viscosity solutions of HamiltonJacobi equations revisited, J. Math. Soc. Japan, 39, 4 (1987), 581-596.

[49] M. G. CRANDALL, M. KOCAN, P. L. LIONS AND A. ŚWIECH, Existence results for boundary value problems for uniformly elliptic and parabolic fully nonlinear equations, Electron. J. Differential Equations, 24 (1999), 1-20.

[50] M. G. CRANDALL, M. KOCAN, AND A. ŚWIECH, $L^{p}$-theory for fully nonlinear uniformly parabolic equations, Comm. Partial Differential Equations, 25, 11-12 (2000), 1997-2053.

[51] M. G. CRANDALL, M. KOCAN, P. SORAVIA, AND A. ŚWIECH, On the equivalence of various weak notions of solutions of elliptic PDE's with measurable ingredients, Progress in Elliptic and Parabolic PDE's (A. Alvino et al. eds.), Pitman Research Notes in Math., 350 (1996), 136-162.

[52] M. G. CRANDALL, P. L. LIONS, Viscosity solutions of Hamilton-Jacobi equations, Trans. Amer. Math. Soc., 277, 1 (1983), 1-42.

[53] M. G. CRANDALL, P. L. LIONS, On existence and uniqueness of solutions of Hamilton-Jacobi equations, Non-Linear Analysis Archive, 10, 4 (1986), 353-370.

[54] M. G. CRANDALL, P. H. RABINOWITZ AND L. TARTAR, On a Dirichlet problem with a singular nonlinearity, Commun. Part. Diff. Eq.,2, 2 (1977), 193-222.

[55] A. CUTRI, F. LEONI, On the Liouville property for fully nonlinear equations, Annales de 1 'institute Henri Poincaré (C) Analyse Non linéaire, 17, 2 (2000), 219-245.

[56] G. DÁVILA, P. FELMER, AND A. QUAAS, Alexandroff-Bakelman-Pucci estimate for singular or degenerate fully nonlinear elliptic equations, C.R. Math., 347, 19-20 (2009), 1165-1168.

[57] G. DÁVILA, P. FELMER AND A. QUAAS, Harnack inequality for singular fully nonlinear operators and some existence results, Calc. Var., 39, 3 (2010), 557-578.

[58] E. N. DANCER, On the Dirichlet problem for weakly non-linear elliptic partial differential equations, Proc. Roy. Soc. Edinburgh Sect. A : Sec. A Math. 76, 4 (1977), 283-300.

[59] D. G DE FIGUEIREDO, P.L. LIONS, AND R.D. NUSSBAUM, A priori estimates and existence of positive solutions of semilinear elliptic equation, J. Math. Pures et Appl., 61, (1982), 41-63.

[60] G. DE PHILIPPIS AND A. FIGALLI, The Monge-Ampère equation and its link to optimal transportation, Bull. Amer. Math. Soc., 51, 4 (2014) 527-580.

[61] K. DEIMLING, Nonlinear Functional Analysis, Dover Edition, 2010.

[62] M. A. DEL PINO, A global estimate for the gradient in a singular elliptic boundary value problem, Proc. Roy. Soc. Edinburgh, Sect. A, 122, 3-4 (1992), 341-352.

[63] L. ESCAURIAZA, $W^{2, n}$ a priori estimates for solutions to fully nonlinear elliptic equations, Indiana Univ. Math. J., 42, 2 (1993) 413-424.

[64] L. C. EVANS, On solving certain nonlinear partial differential equations by accretive operator methods, Israel J. Math., 36, 3-44 (1980), 225-247.

[65] L. C. EVANS, Classical solution of fully nonlinear, convex, second-order elliptic equation, Comm. Pure Appl. Math., 35, 3 (1982), 333-363.

[66] L. C. EVANS, A convergence theorem for solutions of nonlinear second-order elliptic equations, Indiana Univ. Math. J., 27, (1978), 875-887.

[67] L. C. EVANS, J. SPRUCK, Motion of level sets by mean curvature, Journal Diff. Geom., 33, 3 (1991), 635-681.

[68] L. C. EVANS, P. E. SOUGANIDIS, Differential games and representation formulas for solutions of Hamilton-Jacobi-Isaacs equations, Indiana Univ. Math. J., 33, 5 (1984), 773-797. 
[69] E. B. FABES, D. W. STROOCK, The $L^{p}$-integrability of Green's functions and fundamental solutions for elliptic and parabolic equations, Duke Math. J., 51, 4 (1984), 997-1016.

[70] P. FELMER, H. CHEN, On Liouville type theorems for fully nonlinear elliptic equations with gradient term, J. Differential Equations, 225, 8 (2013), 2167-2195.

[71] P. FELMER, A. QUAAS, Fundamental solutions and two properties of elliptic maximal and minimal operators, Trans. Amer. Math. Soc., 361, 11 (2009), 5721-5736.

[72] P. L. FELMER, A. QUAAS, Positive radial solutions to semilinear equation involving the Pucci's operator, J. Differential Equations, 199, 2 (2004), 376-393.

[73] P. Felmer, A. Quaas, Some Recent Results on Equations Involving the Pucci's Extremal Operators, Progr. Nonlinear Differential Equations Appl., 66, (2006), 263-281.

[74] P. FELMER, A. QUAAS, On Critical exponents for the Pucci's extremal operators, Ann Inst. Henri Poincare, Analyse non lineaire 20, 5 (2003), 843-865.

[75] P. FELMER, A. QUAAS, Critical exponents for uniformly elliptic extremal operators, Indiana Univ. Math Journal, 552 (2006), 593-629.

[76] P. FELMER, A. QUAAS AND B. SIRAKOV, Resonance phenomena for second-order stochastic control equations, SIAM J. Math. Anal., 42, 3 (2010), 997-1024.

[77] P. FELMER, A. QUAAS AND B. SIRAKOV, Landesman-Lazer type results for second order Hamilton-Jacobi-Bellman equations, J. Funct. Anal., 258, 12 (2010), 4154-4182.

[78] P. FELMER, A. QUAAS AND B. SIRAKOV, Existence and regularity results for fully nonlinear equations with singularities, Math. Ann. 354, 1 (2012), 377-400.

[79] W. H. FLEMING, R. W. RISHEL, Deterministic and stochastic optimal control, Springer-Verlag, New York Inc. 1975.

[80] P. FOK, Some maximum principles and continuity estimates for fully nonlinear elliptic equations of second order, Ph.D. Thesis, UCSB, 1996.

[81] F. DA LIO, B. SIRAKOV, Symmetry results for viscosity solutions of fully nonlinear uniformly elliptic equations, J. Eur. Math. Soc., 9, 2 (2007), 317-330.

[82] G. GALISE, Maximum principles entire solutions and removable singularities of fully nonlinear second order equations, Math PhD, Facolta di Scienze Matematiche, Fisiche e Naturali, Dipartimento di Matematica, Universita degli Studi di Salerno, 2012.

[83] B. GIDAS, J. SPRUCK, Global and local behavior of positive solutions of nonlinear elliptic equations, Comm. Pure Appl. Math., 34, 4 (1981), 525-598.

[84] B. GIDAS, J. SPRUCK, A priori bounds for positive solutions of nonlinear elliptic equations, Comm. Partial Differential Equations, 6, 8 (1981), 883-901.

[85] D. GILBERG, N. S TRUDINGER, Elliptic partial differential equations of second order, Berlin Heidelberg New York, Reprint of the 2nd ed. 1983.

[86] C. GUI, F. H. LIN, Regularity of an elliptic problem with a singular nonlinearity, Proc. Roy. Soc. Edinburgh Sect. A, 123, 6 (1993), 1021-1029.

[87] C. E. GUTIERREZ, The Monge-Ampère Equation (Progress in Nonlinear Differential Equations and Their Applications) Birkhäuser, 2001.

[88] Q. HAN, N. NADIRASHVILI AND Y. YUAN, Linearity of homogeneous order-one solutions to elliptic equations in dimension three, Comm. Pure Appl. Math, 56, 4 (2003), 425-432.

[89] P. HESS, On a theorem by Landesman and Lazer, Indiana Univ. Math. J., 23 (1974), 827-829.

[90] C. IMBERT, Alexandroff-Bakelman-Pucci estimate and Harnack inequality for degenerate/singular fully non-linear elliptic equations, J. Differential Equations, 250, 3 (2011), 1553-1574.

[91] C. IMBERT, L. SILVESTRE, Estimates on elliptic equations that hold only where the gradient is large, arXiv:1306.2429v2 [math.AP], (2013), 1-15.

[92] R ISAACS, Games of pursuit Rand Corp, Report No.(1951) 1-257.

[93] R ISAACS, Differential Games, Wiley, New York, 1965.

[94] H. ISHII, On uniqueness and existence of viscosity solutions of fully nonlinear second-order elliptic PDE's, Comm. Pure Appl. Math, 42, 1 (1989), 15-45.

[95] H. ISHII, Perron's method for Hamilton-Jacobi equations, Duke Math. J., 55, 2 (1987), 369-384.

[96] H. ISHII, Remarks on existence of viscosity solutions of Hamilton-Jacobi equations, Bull. Fac. Sci. Engng. Chuo Univ., 26, (1983), 5-24.

[97] H. ISHII, Existence and uniqueness of solutions of Hamilton-Jacobi equations, Funkcial Ekvac., 29, (1986), 167-188. 
[98] H. ISHII, P. L LIONS, Viscosity solutions of fully nonlinear second-order elliptic partial differential equations, J. Differential Equations, 83, 1 (1990), 26-78.

[99] R. JENSEN, The Maximum principle for viscosity solutions of fully nonlinear second order partial differential equations, Arch. Rat. Mech. Anal., 101, 1 (1988), 1-27.

[100] R. JENSEN, P.L LIONS AND P.E SOUGANIDIS, A uniqueness result for viscosity solutions of second order fully nonlinear partial differential equations, Proc. Amer. Math. Soc., 102, 4 (1988), 975978.

[101] P. JUUTINEN, P. LINDQUIST AND J. MANFREDI, On the equivalence of viscosity solutions and weak solutions for a quasi linear equation, SIAM J. Math. Anal., 33, 3 (2001), 699-717.

[102] N. KATZOURAKIS, An introduction to viscosity solutions for fully nonlinear PDE with applications to calculus of variations in $L^{\infty}$, Springer Briefs in Mathematics, 2015.

[103] S. KOIKE, A beginner's guide to the theory of viscosity solutions, The Mathematical Society of Japan, Tokyo, 2014.

[104] S. KOIKE, A. ŚWIECH, Maximum principle and existence of $L^{p}$-viscosity solutions for fully nonlinear uniformly elliptic equations with measurable and quadratic term, NoDEA Appl., 11, 4 (2004), 491-509.

[105] S. KOIKE, A. ŚWIECH, Existence of strong solutions of Pucci extremal equations with superlinear growth in $D u$, Journal of Fixed Point Theory and Applications, 5, 2 (2009), 291-304.

[106] J. KOVATS, The minimax principle and $W^{2, p}$ regularity for solutions of the simplest Isaacs equations, Proc. Amer. Math. Soc., 140, 8 (2012), 2803-2815.

[107] J. KOVATS, Probabilistic solutions of the Dirichlet problem for Isaacs equation, Hindawi Publishing Corporation Proceedings of the Conference on Differential and Difference Equations and Applications, 593-604.

[108] N. V. KRYLOV, Control of a solution of a stochastic integral equation, Theory Probab. Appl., 17, 1 (1972), 114-130.

[109] N. V. KRYLOV, On control of the solution of a stochastic integral equation with degeneration, Izv. Akad. Nauk SSSR Ser. Mat., 36, 1 (1972), 248-261.

[110] N. V. KRYLOV, Controlled diffusion process, Springer-Verlag, Berlin-Heidelberg-New York, (1980).

[111] N. V. KRYLOV, On nonlinear elliptic equations in a domain, Uspekhi Mat. Nauk, 226 37-4 (1982).

[112] N. V. KRYLOV, Boundedly nonhomogeneous elliptic and parabolic equations, Izv. Akad. Nauk SSSR Ser. Mat., 46, 3 (1982), 487-523.

[113] N. V. KRYLOV AND M. V. SAFONOV, An estimate of the probability that a diffusion process hits a set of positive measure, Dokl. Akad. Nauk SSSR., 245, 1 (1979), 18-20.

[114] N. V. KRYLOV, M. V. SAFONOV, A certain property of solutions of parabolic equations with measurable coefficients, Izv. Akad. Nauk SSSR Ser. Mat., 44, 1 (1980), 161-175.

[115] H. J. KUO, N. S. TRUDINGER, Discrete methods for fully nonlinear elliptic equations, SIAM J. Numer. Anal., 291 (1992), 123-135.

[116] H. J. KUSHNER, Stochastic Stability and Control, Academic Press, 1967.

[117] F. LEONI, Explicit subsolutions and a Liouville theorem for fully nonlinear uniformly elliptic inequalities in halfspaces, Journal de Mathmatiques Pures et Appliquèes, 98, 5 (2012), 574-590.

[118] C. LI, Monotonicity and symmetry of solutions of fully nonlinear elliptic equations on unbounded domains, Comm. Partial Differential Equations, 16, 4 (1991), 585-615.

[119] F. H. LIN, Second derivative Lp-estimates for elliptic equations of nondivergent type, Proc. Amer. Math. Soc, 96, 3 (1986), 447-451.

[120] P. L. LIONS, On the Hamilton-Jacobi-Bellman equations, Acta Appl. Math., 1, 1 (1983), 17-41.

[121] P. L. LIONS, Existence results for First-order Hamilton-Jacobi equations, Ricerche Mat., 32, 1 (1983), $3-23$.

[122] P. L. LIONS, Optimal control of diffusion processes and Hamilton-Jacobi-Bellman equations, Part 1: The dynamic programming principle and applications, to appear in Comm. P.D.E., 8, 10 (1983), 1101-1174.

[123] P. L. LIONS, Optimal control of diffusion processes and Hamilton-Jacobi-Bellman equations part 2 : viscosity solutions and uniqueness, Comm. Partial Differential Equations, 8, 11 (1983), 1229-1276.

[124] P. L LIONS, Viscosity solutions of fully nonlinear second-order equations and optimal stochastic control in infinite dimensions Part III. Uniqueness of viscosity solutions for general second-order equations, J. Funct. Anal., 86, 1 (1989), 1-18.

[125] P. L. LIONS, Bifurcation and optimal stochastic control, Nonlinear Anal., 7, 2 (1983), 177-207. 
[126] P.L. LIONS, Generalized solutions of Hamilton-Jacobi equations," Research notes in mathematics, Boston-London-Melbourne: Pitman Advanced Publishing Program, 69, (1982).

[127] P.L. LIONS, Two remarks on Monge-Ampère equations, Ann. Mat. Pura Appl., 142, 4 (1985), 263275.

[128] P. L. LIONS, Viscosity solutions of fully nonlinear second-order equations and optimal stochastic control in infinite dimensions, Part I: the case of bounded stochastic evolutions, Acta Mathematica., 161, 1 (1988), 243-278.

[129] P. L. LIONS, Optimal control of diffusion processes and Hamilton-Jacobi-Bellman equations, Part III. Regularity of the optimal cost function, Nonlinear Partial Differential Equations and Their Applications, College de France Seminar, Vol. V, Res. Notes Math., 93, (1981-1982), 95-205.

[130] P. L. LIONS, P. E. SOUGANIDIS, Viscosity solutions of second-order equations stochastic control and stochastic differential games, Stochastic Differential Systems, Stochastic Control Theory and Applications The IMA Volumes in Mathematics and Its Applications, 10, (1988), 293-309.

[131] G. LU, J. ZHU, Liouville-type theorems for fully nonlinear elliptic equation in half spaces, arXiv:1209.1144v2 [math.AP].

[132] M. BADIALE, Geometrical properties of fully nonlinear equations and an application to singularities, J. Differential Equations, 112, 1 (1994), 33-52.

[133] J.H.MICHAEL, Barriers for uniformly elliptic equations and exterior cone condition, J. Math. Anal. Appl., 79, 1 (1981), 203-217.

[134] K. MILLER, Barriers on cones for uniformly elliptic operators, Ann. Mat. Pura Appl., 76, 1 (1967), 93-106.

[135] K. MILLER, Extremal barriers on cones with Phragmen-Lindelof theorems and other applications, Ann. Mat. Pura Appl., 90, 1 (1971), 297-329.

[136] N. NADIRASHVILI, Y. YUAN, Homogeneous solutions to fully nonlinear elliptic equation, Proc. Amer. Math. Soc., 134, 6 (2006), 1647-1649.

[137] N. NADIRASHVILI, S. VLĂDUT, Nonclassical solutions of fully nonlinear elliptic equations, GAFA, Geom. Funct. Anal., 17, 4 (2007), 1283-1296.

[138] N. NADIRASHVILI, S. VLĂDUT, Singular solutions of Hessian fully nonlinear elliptic equations, Adv. Math., 228, 3 (2011), 1718-1741.

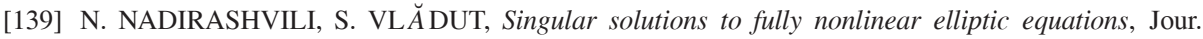
Math. Pures Appl., 89 (2008), 107-113.

[140] N. NADIRASHVILI, S. VLĂDUT, Homogeneous solutions of fully nonlinear elliptic equations in four dimensions, Commun. Pure Appl. Math., 66, 10 (2013), 1653-1662.

[141] N. NADIRASHVILI, V. TKACHEVB AND S. VLĂ DUT, A non-classical solution to a Hessian equation from Cartan isoparametric cubic, Advances in Mathematics, 231, 3-4 (2012), 1589-1597.

[142] L. NIRENBERG, On nonlinear elliptic partial differential equations and Hölder continuity, Comm. Pure Appl. Math. 6, 1 (1953), 103-156.

[143] L. NIRENBERG, On a generalization of quasi-conformal mappings and its application to elliptic partial differential equations, Contributions to the Theory of Partial Differential Equations, edited by L. Bers et al., Ann. of Math., Princeton Univ. Press, Princeton, N.J., 33, (1954) 95-100.

[144] A. V. POGORELOV, The Dirichlet problem for the n-dimensional analog of the Monge-Ampére equation, Dokl. Akad. Nauk 201 (1971), 790-793.

[145] M. H. PROTTER, H. F. WEINBERGER, Maximum principles in differential equations, Springer New York, 1999.

[146] C. PUCCI, Operatori ellittici estremanti, Ann. Mat. Pure Appl., 72,1 (1966), 141-170.

[147] C. PUCCI, Maximum and minimum first eigenvalues for a class of elliptic operators, Proc. Amer. Math. Soc., 17, 4 (1966), 788-795.

[148] A. QUAAS, Existence of positive solutions to a semilinear equation involving the Pucci's operator in a convex domain Diff. Integral Equations, 17, 5-6 (2004), 481-494.

[149] A. QUAAS, B. SIRAKOV, Existence results for nonproper elliptic equations involving the Pucci operator, Comm. Part. Diff. Eq., 31, 7 (2006), 987-1003.

[150] A. QUAAS, B. SIRAKOV, Principal eigenvalues and the Dirichlet problem for fully nonlinear elliptic operators, Adv. Math., 218. 1 (2008), 105-135.

[151] P. RABINOWITZ, Theorie du degre topologique et applications a des problemes aux limites non lineares, Lectures Notes Lab, Analyse Numerique Universite Paris, Vi 1975. 
[152] M. V. SAFONOV, Non-divergence elliptic equations of second order with unbounded drift, In Nonlinear Partial Differential Equations and Related Topics, AMS Transl., 229, 2 (2010), 211-232.

[153] O. SAVIN, Small perturbation solutions for elliptic equations, Comm. Partial Differential Equations, 32, 4 (2007), 667-578.

[154] B. SIRAKOV, Nonuniqueness for the Dirichlet problem for fully nonlinear elliptic operators and the Ambrosetti-Prodi phenomenon, Progr. Nonlinear Differential Equations Appl., 85, (2014), 405-421.

[155] B. SIRAKOV, Solvability of uniformly elliptic fully nonlinear PDE, Arch. Rational Mech. Anal., 195, 2 (2010), 579-607.

[156] A. ŚWIECH, $W^{1, p}$-interior estimates for solutions of fully nonlinear uniformly elliptic equations, Adv. Differential Equation, 2, 6 (1997), 1005-1027.

[157] N. S. TRUDINGER, Hölder gradient estimates for fully nonlinear equations, Proceedings of the Royal Society of Edinburgh, Section A, 108, 1-2 (1988), 57-65.

[158] N. S. TRUDINGER, Comparison principles and pointwise estimates for viscosity solutions of nonlinear elliptic equations, Rev. Mat. Iberoam., 4, 3-4 (1988), 453-468.

[159] N. S. TRUDINGER, On regularity and existence of viscosity solutions of nonlinear second order elliptic equations Partial Differential Equations and the Calculus of Variations, II, Progr. Nonlinear Differential Equations Appl., Birkhauser Boston, Boston, 2, (1989), 939-957.

[160] N. S. TRUDINGER, X. J. WANG, The Monge-Ampère equation and its geometric applications, Handbook of Geometric Analysis, International Press, I, (2008), 467-524.

[161] J. I. E. URBAS, Elliptic equations of Monge-Ampère type, Thesis, Australian Nat. Univ., Canberra, 1984.

[162] L. WANG, On the regularity theory of fully nonlinear parabolic equations I, Comm. Pure Appl. Math., 45, 1 (1992), 27-76.

[163] L. WANG, On the regularity theory of fully nonlinear parabolic equations, II Comm. Pure Appl. Math., 45, 2 (1992), 141-178.

[164] J. WIGNIOLLE, A strong maximum principle for fully nonlinear degenerate operators, Prépublications de l'université de Cergy-Pontoise.

[165] N. WINTER, $W^{1, p}$ and $W^{2, p}$-estimates at the boundary for solutions of fully nonlinear, uniformly elliptic equations, Z. Anal. Anwend., 28, 2 (2009), 129-164. 